\title{
EBG Structures for Reduction of Mutual Coupling in
}

\section{Patch Antennas Arrays}

\author{
F. Benykhlef, and N. Boukli-Hacene
}

\begin{abstract}
An important issue in antenna array design is reduction of mutual coupling. In square microstrip antennas this reduction can be achieved by using electromagnetic band-gap (EBG) structures. They can help in the reduction of mutual coupling by using their capability of suppressing surface waves propagation in a given frequency range. In this paper, we analyze the isolation properties of different EBG structures are compare them in antennas arrays by simulations. A new configuration of a planar compact electromagnetic bandgap structure is investigated. Compared to the conventional EBG (mushroom structure), a size reduction of $67.2 \%$ is achieved. Simulation results show that a significant value of mutual coupling reduction, more than $6 \mathrm{~dB}$, can be obtained by using the proposed structure.
\end{abstract}

Index Terms----Electromagnetic-bandgap (EBG), mutual coupling, structures, microstrip antenna arrays, surface wave.

\section{INTRODUCTION}

With the drastic demand of wireless communication system and their miniaturization, antenna design becomes more challenging. Recently patch antennas have been widely used. In spite of its several advantages, as light weight and low volume, low profile, planar configuration which can be easily made conformal to host surface, low fabrication cost, and the capability of obtaining dual and triple frequency operations.[1][2]

On the other hand, when the antenna is operating in the fundamental mode (TM10 for rectangular patches), surface waves are strongly excited in E-plane. In this mode, the field distribution can excite the TM0 mode of the surface waves $\mathrm{E}$ plane [3]. If multiple antennas share the same ground plane, surface currents can cause unwanted mutual coupling.

Mutual coupling in arrays is considered to be very import problem for antenna design engineers. In arrays of microstrip antennas, this coupling is especially critical. This coupling can be due to radiated waves or due to surface waves [3]. Surface waves can become dominant if high dielectric constant substrates are used [4]. To overcome these limitations of microstrip patch antennas, electromagnetic band gap (EBG)

Manuscript received November 22, 2016; revised February 15, 2017 and February 22, 2017. Date of publication March 28, 2017.

Authors are with the University of Tlemcen, Faculty of Technology, telecommunication laboratory, BP: 230, 13000 Chetouane, Algeria.

E-mails: benyakhlefttl@outlook.com, bouklin@yahoo.com Digital Object Identifier (DOI): 10.24138/jcomss.v13i1.242 structures are used. The main property of EBG structures is suppression of surface wave propagation in a specific frequency band [5][6]. Due to the capability of these structures for reducing the surface waves, they can improve the antenna performance. This improvement can be achieved in reduction of back-lobe radiation, increasing the antenna gain and increasing the antenna efficiency [7].

Different forms of EBG structures such as mushroom-like EBG structure or dielectric rods and holes are studied and evaluated their capability to reduce mutual coupling effect. We propose a new, compact and easy to fabricate, EBG structure, based on totally planar layout without via. Bandgap feature of the EBG structure is studied using dispersion diagram and performance of two mutually coupled patch antennas.

This paper is organized as follows. The 2D EBG gap has been studied and is inserted between two square patch antennas (operating at $2.4 \mathrm{GHz}$ ) on a tick and highpermittivity substrate to reduce coupling mutual effect. After that, we replaced the $2 \mathrm{D}-\mathrm{EBG}$ with mushroom EBG structure and we evaluated the isolation between antennas.

Secondly, three HIS structures (fork, mushroom and proposed planar structure) on a thin and low dielectric constant substrate $\left(\varepsilon_{y}=2.5, \mathrm{~h}=1.588 \mathrm{~mm}\right)$ are analyzed and are inserted between rectangular patch antenna (operating at $10 \mathrm{GHz}$ ).

The simulations are conducted using Ansoft HFSS ${ }^{\text {TM }}$ based on the finite element method (FEM) and MATLAB ${ }^{\circledR}$ program based on the plane wave method (PWM).

\section{BAND GAP CHARACTERIZATION OF THE 2D-EBG STRUCTURE}

The EBG structure should be designed so that the antenna operating frequency should be in located at the center of the bandgap. The 2D-EBG photonic crystal substrate are introduced to attenuate surface wave modes.

The idea is to design a square patch antenna on a $2 \mathrm{D}$ photonic crystal substrate, where the patch becomes the "defect" in the EBG structure.

In order for the band gap to exist, the substrate (host medium) to impurity (posts) refractive index ratio must be greater than 2:1 (or dielectric constant ratio must be greater than 4:1) [8].

Thus, to obtain the broadest band gap for $\varepsilon_{r s}=10$ air posts $\varepsilon_{r p}=1$ are used. For the sake of simplicity, the square lattice form is used. The dispersion diagrams for different values of 
the filling factor $\left(R_{c} / a\right)$ are illustrated in Figure 1 (here $R_{c}$ is the radius of the cylindrical air posts and $(a)$ is the lattice constant). These diagrams reveal the band gaps for both TE and TM polarizations and help to determine the appropriate lattice dimensions.

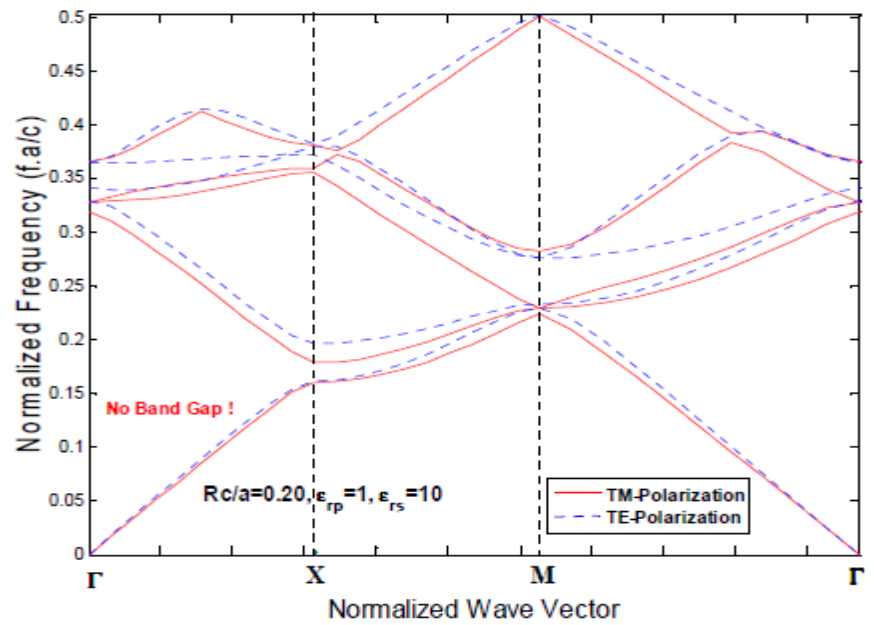

(a)

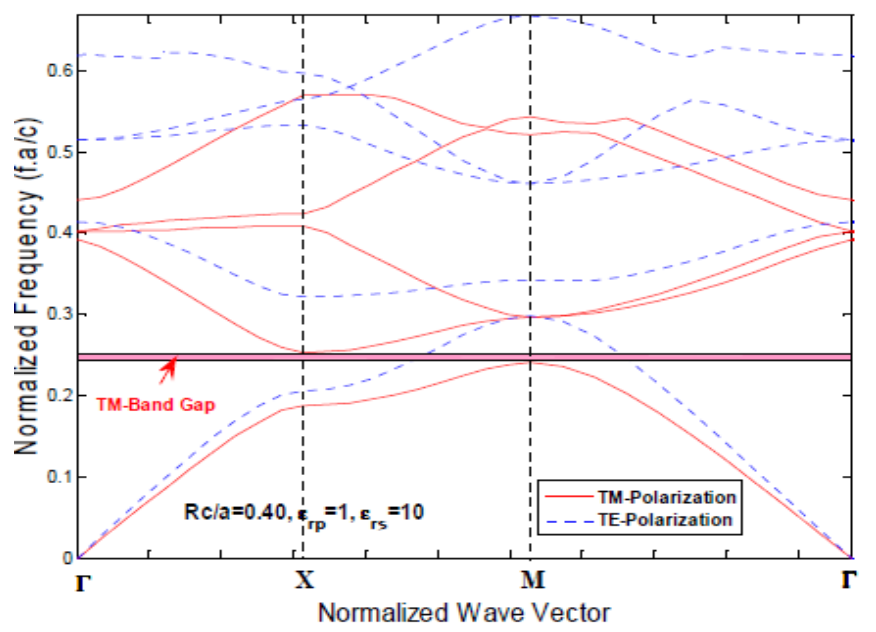

(b)

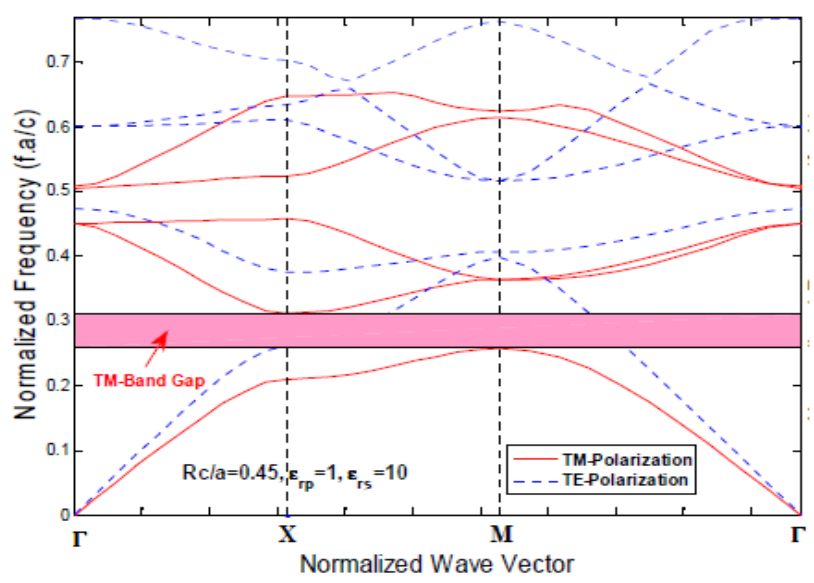

(c)

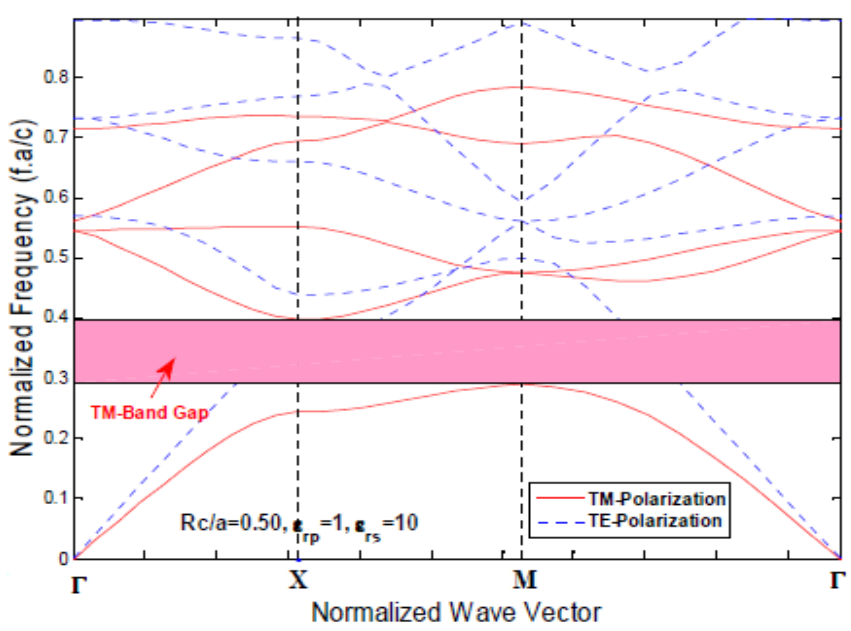

(d)

Fig. 1. The dispersion diagrams for different filling factors (a) for 0.20 (b) for 0.40 (c) for 0.45 (d) for 0.50. In MATLAB

Investigation of the above diagrams show that no band gaps appear for small values of the filling factor. However, when the filling factor gets higher than 0.40 , more significant band gaps appear. Therefore, the study is restricted for $0.4<\frac{R_{\varepsilon}}{a}<0.5$. To fit the desired requirements (low lattice constant, and wide Bandgap), $\left(\frac{R_{c}}{a}=0.48\right)$ is selected which gives:

TABLE I

THE SELECTED DIMENSIONS TO DESIGN THE SUITABLE EBG STRUCTURE

\begin{tabular}{ccccc}
\hline$\left(\boldsymbol{R}_{\boldsymbol{c}} / \boldsymbol{a}\right)$ & $\mathbf{a}(\mathbf{m m})$ & $\boldsymbol{R}_{\boldsymbol{c}}(\mathbf{m m})$ & $\boldsymbol{f}_{\boldsymbol{N}}$ & $\begin{array}{c}\text { BGL } \\
(\mathbf{M H z})\end{array}$ \\
\hline 0.48 & 19.164 & 39.925 & 0.3194 & 665 \\
\hline
\end{tabular}

The dispersion diagrams that characterize our found EBG structure are shown in Figure 2.

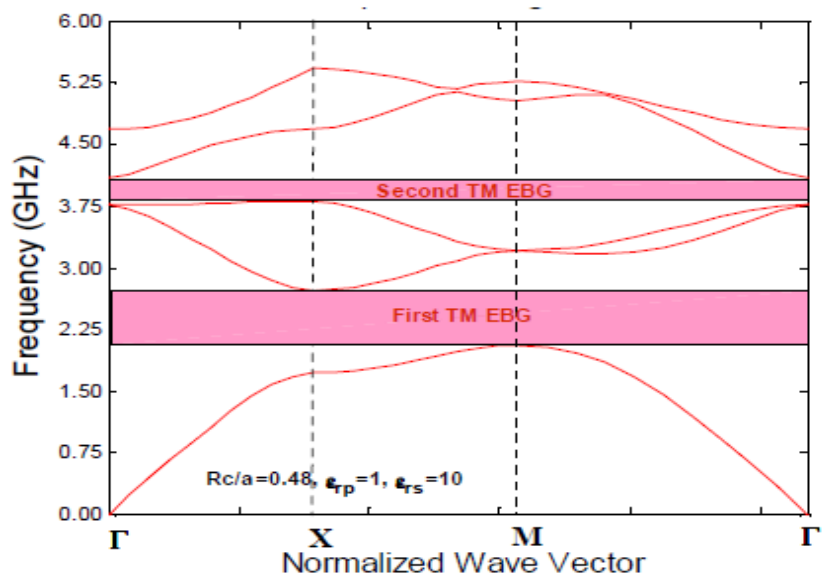

Fig. 2. The dispersion diagrams of the designed EBG structure TM polarization 


\section{DESIGN AND Simulations OF EBG ANTENNAS}

The EBG antenna is designed to operate at the frequency $2.4 \mathrm{GHz}$. It is excited by a coaxial probe and the feed point is located at the distance $(\mathrm{dx}=2.7 \mathrm{~mm}, \mathrm{dy}=0)$ away from the edge of the patch. The length $\mathrm{L}$ and the width $\mathrm{W}$ have been taken as $16.38 \mathrm{~mm}$, six EBG rows of holes are drilled in the substrate. The substrate thickness is chosen to be: $6 \mathrm{~mm}$ (thick) to ensure favorable conditions for the excitation of surface waves $\left(\frac{\hbar}{\lambda_{0}}=0.048>0.03\right)$, the dielectric material Arlon AR1000 with the dielectric constant 10 with dissipation factor 0.003 is chosen due to the higher excitation of surface waves.

The reflection coefficients for both microstrip antennas with and without EBG are depicted in Figure 3.

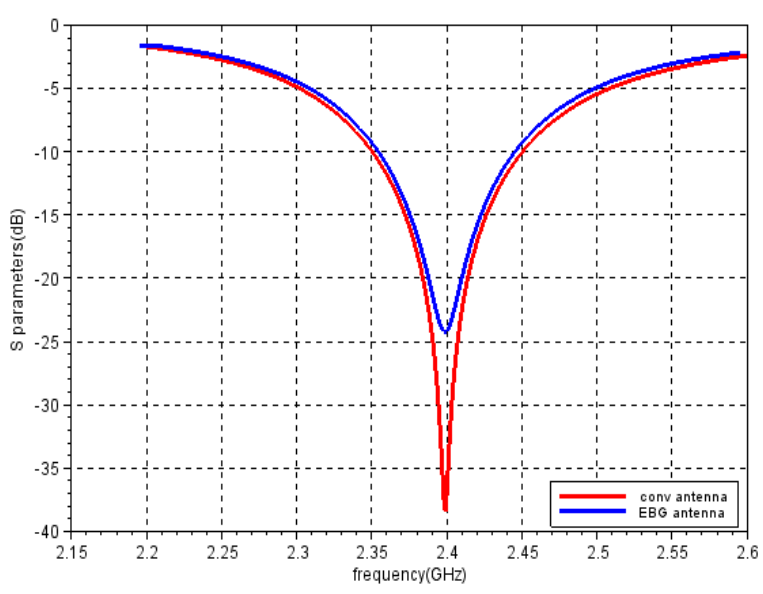

Fig. 3. Return losses of the patch antenna with and without EBG.

The simulation results show that the reflection coefficient minimum level (at $\mathrm{f}=2.4 \mathrm{GHz}$ ) increases $47 \%$ from $-38.40 \mathrm{~dB}$ for MPA without EBG to $-24.25 \mathrm{~dB}$ for MPA with EBG.

This increase is due to the insertion of the photonic crystal that increases the reflection of surface waves to the excitation source.

\section{Mutual COUPLing Reduction}

The two patch antennas are placed $0.75 \lambda_{0} \mathrm{~mm}$ (edge to edge) apart from each other. Two rang of 2D-EBG structures are inserted between them to reduce Mutual Coupling between the radiating elements. To investigate the proposed antenna design shown in Figure 4, high frequency structure simulation (HFSS) software are used for simulation and optimization.

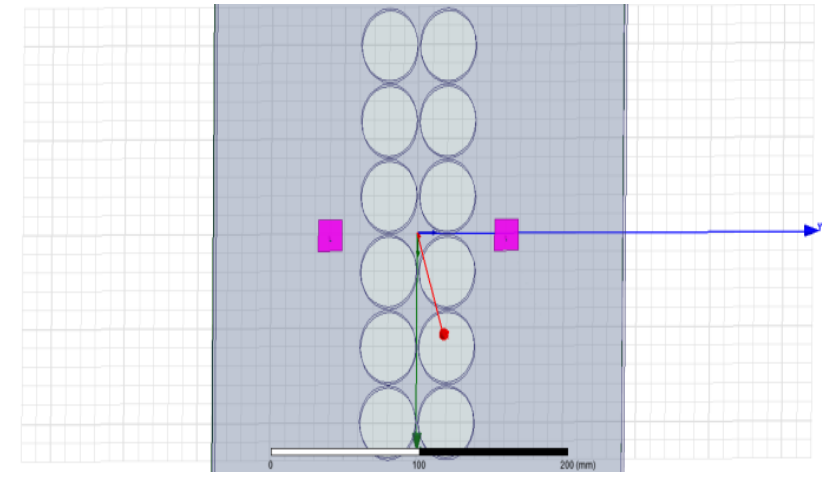

Fig. 4. Array of two patches in dielectric substrate including a 2DEBG

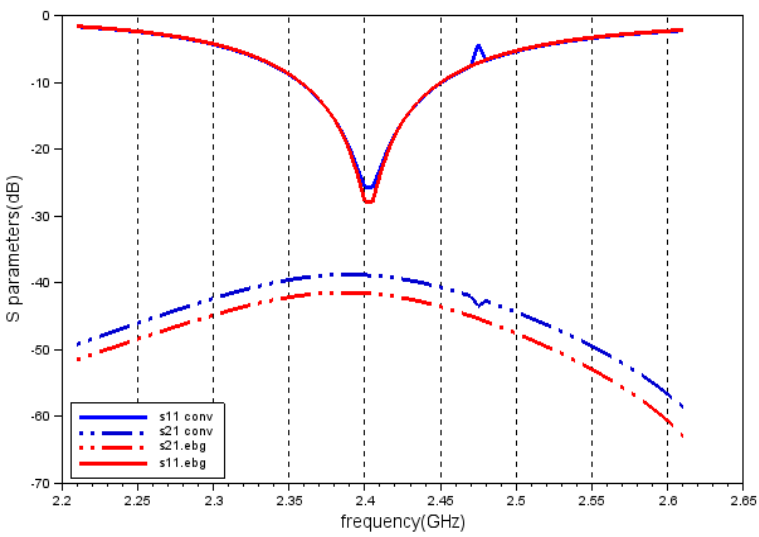

Fig. 5. Computed results of microstrip antennas with and without the EBG structure

The computed results by using HFSS $^{\mathrm{TM}}$ are shown in (Figure 5). The return loss of both antenna is $-28 \mathrm{~dB}$ at the resonate frequency. The mutual coupling of the antennas without the EBG structure is $-39 \mathrm{~dB}$. In comparison, the mutual coupling of the patches with the EBG structure is only $-41.55 \mathrm{~dB}$. An approximately $2.5 \mathrm{~dB}$ reduction of mutual coupling is achieved.

Now, it is suggested to modify the designed EBG antenna by replacing its ground plane with a suitable well-designed metallodielectric EBG structure as shown in figure 6.

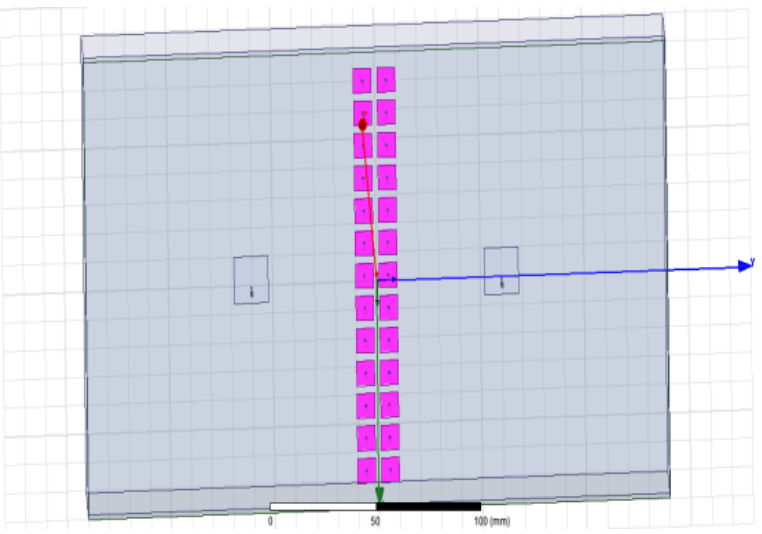

Fig. 6. Array of two patches in high dielectric substrate including a mushroom like EBG structures 
Using HFSS simulation tool, mushroom EBG like is simulated. The Reflection phase characteristic of the optimized EBG structure is show in figure 7.
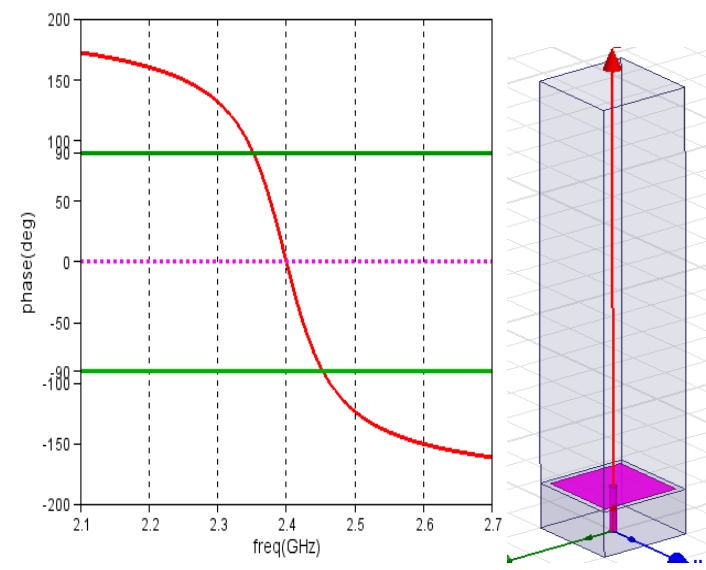

(a)

(b)

Fig. 7. (a) Simulated results of reflection phase for b) EBG structure Mushroom like EBG (simulated model), gap $=1.38 \mathrm{~mm}$, wpatch $=8.62 \mathrm{~mm}$

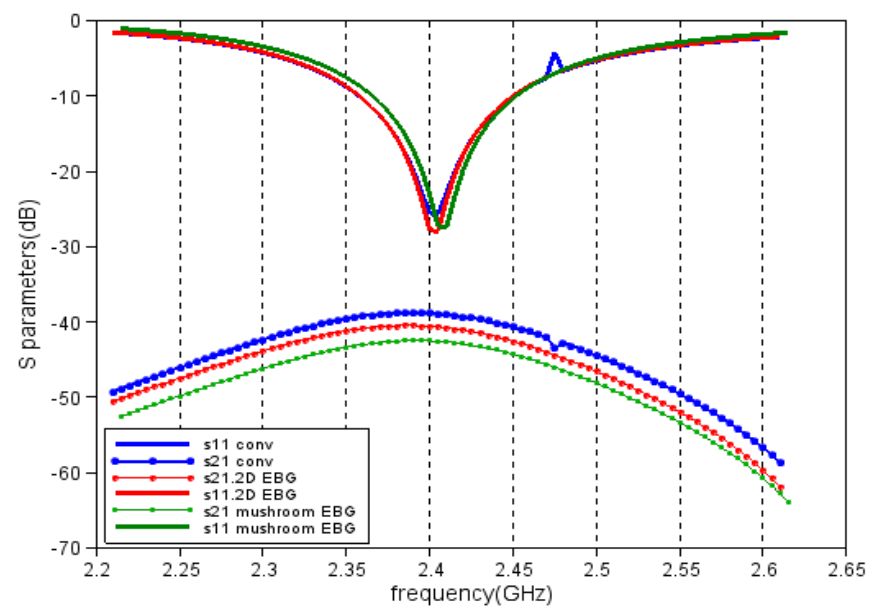

Fig. 8. Computed results of microstrip antennas with and without the EBG structure

As shown in Fig.8, the resonance frequency is $2.4 \mathrm{GHz}$. Frequency band of \pm 90 degree reflection phase is from 2.35 $\mathrm{GHz}$ to $2.45 \mathrm{GHz}$.

It is observed that for the antennas without the EBG structure, the mutual coupling is $-39 \mathrm{~dB}$. In comparison, the mutual coupling of the antennas with the EBG structure with two colon is $42.4 \mathrm{~dB}$, An $4.5 \mathrm{~dB}$ mutual coupling reduction is observed at the resonant frequency.

In figure 8, the results show that mushroom like EBG structure enhance the decoupling between the square patch antenna on a high-permittivity substrate $\varepsilon_{y}=10$ with $2 \mathrm{~dB}$ compared with the $2 \mathrm{D}-\mathrm{EBG}$.

Analysis had showed EBG mushroom structure with appropriate gap and position is useful in reduction of coupling between antenna patches for this reason we used hereafter only the structure HIS (high impedance structure or EBG structure)
In this second part of the article, three HIS structures (fork, mushroom and proposed planar structure) on a thin and low dielectric constant substrate $\left(\varepsilon_{\gamma}=2.5, \mathrm{~h}=1.588 \mathrm{~mm}\right)$ are analyzed using HFSS $^{\mathrm{TM}}$ which is based on the finite element method (FEM).

\section{HIS STRUCTURE CHARACTERIZATION}

The surface impedance of a HIS structure is frequency sensitive since the EBG structure actually forms a distributed $L C$ network with specific resonant frequencies. The capacitance $C$ is determined by the fringing capacitance between neighboring metal patches, while the current as flowing through inductance, can be considered as inductance $L$. The equivalent $L C$ circuit acts as a two-dimensional electric filter to block the flow of the surface waves in the frequency range where the surface impedance is very high. The capacitance and inductances in the equivalent circuit can be approximated by the following formulas [9]:

$$
\begin{gathered}
c=\frac{w \cdot \varepsilon_{0}\left(1+\varepsilon_{0}\right)}{\pi} \cosh ^{-1}\left(\frac{w+g}{g}\right) \\
L=\mu_{0} \mu_{y} h \\
f_{0}=\frac{1}{2 \pi \sqrt{L C}}
\end{gathered}
$$

The fractional bandwidth (BW) of reflection phase between \pm 90 degrees is

$$
B W=\frac{1}{\eta} \sqrt{\frac{L}{C}}
$$

And $\eta=120 \mathrm{Ohm}$ is the free space impedance.

Figure 9 presented the three HIS structures (EBG mushroom, planar proposed EBG and Fork-like patches and the unit cells dimensions.

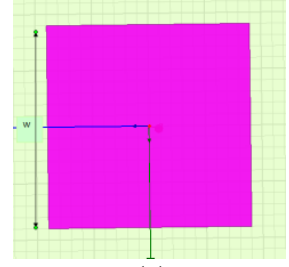

(a)

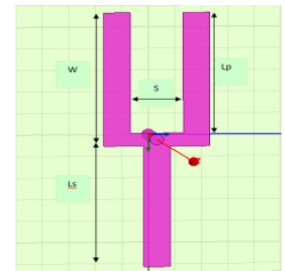

(b)

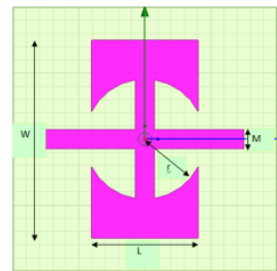

(c)
Fig. 9. The three High impedance Surface (HIS), (a) Mushroom cells (with via) (b) Fork-like cells (with via) and (b) proposed structure (with via)

The dimensions are, (a) $W=3.5 \mathrm{~mm}$, gap $=0.6 \mathrm{~mm}$ (b) $W=3.1 \mathrm{~mm}, \quad L s=L p=2.8 \mathrm{~mm}, s=1 \mathrm{~mm}, d=0.5 \mathrm{~mm}, g a p=0.5$ mm.(c) $W=3 \mathrm{~mm}, \quad \mathrm{M}=0.3 \mathrm{~mm}, \quad \mathrm{r}=0.91 \mathrm{~mm}, \quad \mathrm{~L}=1.62 \mathrm{~mm}$, gap $=0.3 \mathrm{~mm}$.

The Reflection phase characteristic of the three EBG structures are show in figure 10. 

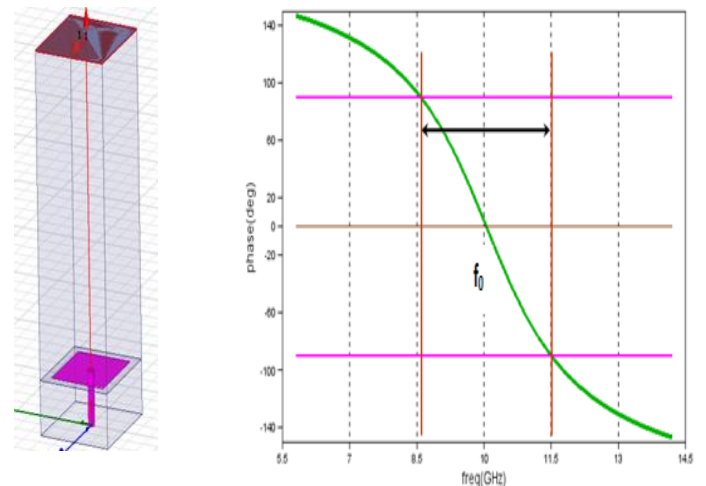

(a)
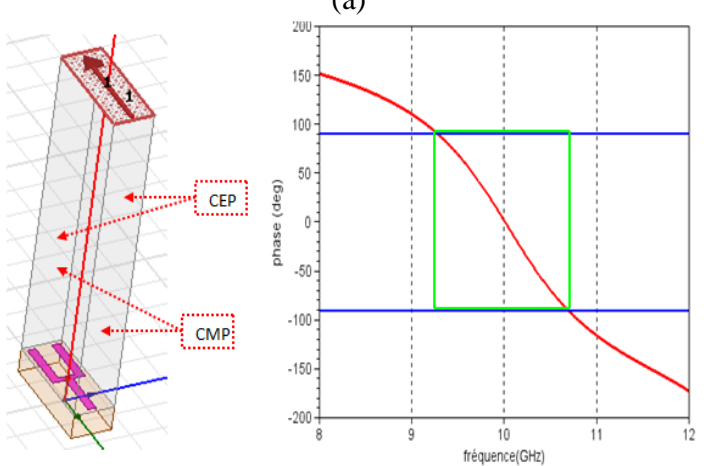

(b)
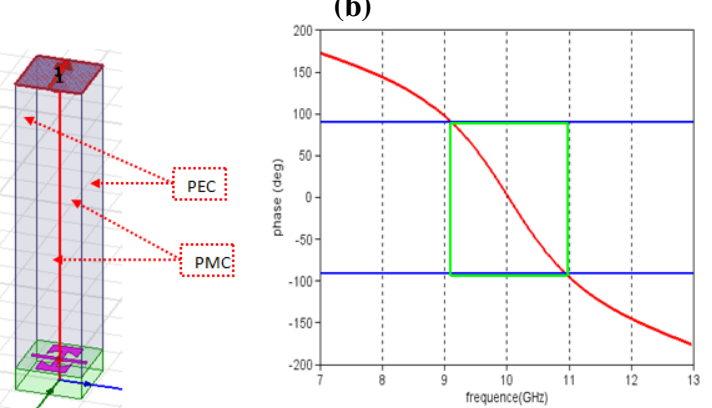

(c)

Fig. 10. Reflection phase of the HIS, (a) the Mushroom-like EBG and (b)the Fork-like EBG (c) proposed structure (with via)

As shown in Fig.8, the simulated reflection phases for both the EBG structures varied continuously from $+180^{\circ}$ to $-180^{\circ}$ relative to the frequency, and were equal to zero degrees at 10 GHz.

The frequency bandwidth that causes the reflection phase response to vary in between $-90 /+90$ degrees gives the reflection band-gap. Operational frequencies and bandwidths from these structures are extracted from the reflection.

We found that the band gap of the three HIS are $31 \%(8.5$ $\mathrm{GHz}-11.5 \mathrm{GHz})$ for the mushroom structure, $15.5 \%(9.25$ $\mathrm{GHz}-10.7 \mathrm{GHz})$ for the fork structure and $19 \%(9.1 \mathrm{GHz}-$ $11 \mathrm{GHz}$ ) for the proposed EBG structure.

\section{REDUCTION OF THE MUTUAL COUPLING BY USING HIS}

An antenna array operating at $10 \mathrm{GHz}$ is designed. To obtain the resonant frequency at $10 \mathrm{GHz}$, the rectangular patch's size was $8.3 \mathrm{~mm} \times 11.34 \mathrm{~mm}$.

The patch antennas are located in the middle of the ground plane with $15 \mathrm{~mm}\left(0.5 \lambda_{0}\right)$ gap between them. A simulation without the EBG structures is used as a reference figure 11.
The focus of the study is the isolation level between the antenna elements, one column of the three different HIS structures was possible to fit between the antennas. The simulated mutual coupling results in E-plane and H-plane configuration are shown in figure 12.

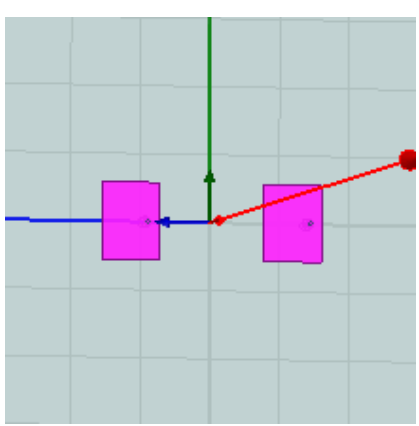

(a)

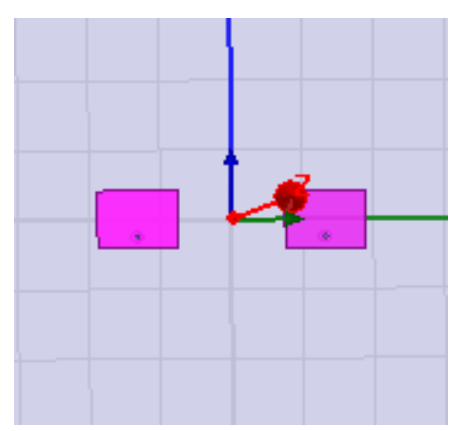

(b)
Fig. 11. Arrays antenna configurations (a) in E plane, (b) in $\mathrm{H}$ plane

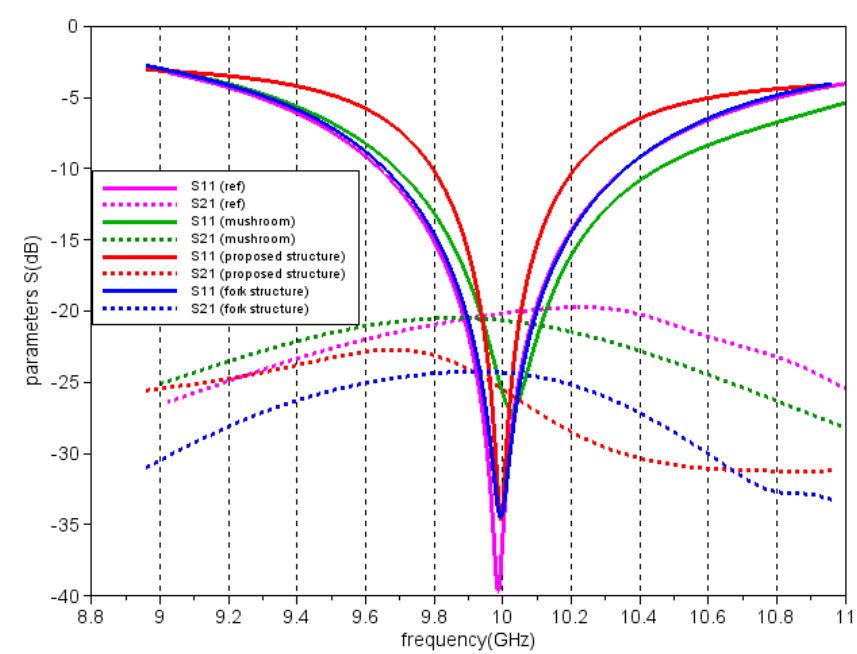

(a)

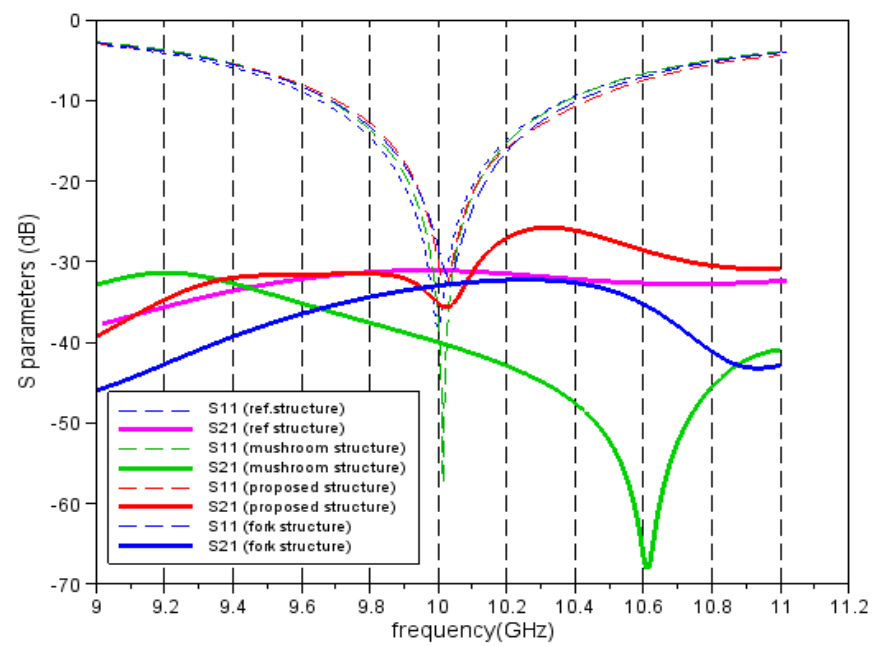

(b)

Fig. 12. Simulated S11 and S12 without and with HIS row. a) E-plan, (b) H-plan 
The achieved mutual coupling effect between the antennas without EBG structures was observed around $-31.8 \mathrm{~dB}$ in $\mathrm{H}-$ plan and $-21.67 \mathrm{~dB}$ in E-plan.

- In E-plane configuration the mutual coupling becomes greater because the centre-to-centre distance is reduced, when the HIS structures were employed, the level of mutual coupling changes. It is clearly noted that when the row of mushroom EBG is inserted; the mutual oupling observed is around $-22.5 \mathrm{~dB}$ at the resonant frequency $10.05 \mathrm{GHz}$. An approximately $4 \mathrm{~dB}$ reduction of mutual coupling is achieved with return loss better than $-10 \mathrm{~dB}$ when the row of fork EBG is inserted; The best attenuation of mutual coupling is achieved with the proposed EBG structure, which according to the simulations has the highest isolation (approximately $6 \mathrm{~dB}$ ). Compared to the mushroom EBG structure, surface waves can be successfully suppressed using proposed EBG structure in E-plan configuration.

- In H-plane configuration, when the fork EBG structure is employed only 2.02-dB reduction is observed. An approximately $6 \mathrm{~dB}$ reduction of mutual coupling is achieved when the row of the proposed EBG structure is inserted. When the EBG structure is employed, a $10.8 \mathrm{~dB}$ mutual coupling reduction is achieved at $10.10 \mathrm{GHz}$, which proves that the surface wave is suppressed. According to simulations, the impedance bandwidth of the antenna elements remained approximately the same with or without the EBG structures of all the cases.

\section{CONCLUSION}

In this paper, the isolation properties of different EBG structures are compared in antennas arrays by simulations.

Firstly, the 2D-EBG concept was applied to the design of a square patch antenna with operating frequency $2.4 \mathrm{GHz}$ to suppress the surface wave propagation in a thick and high dielectric constant substrate $\varepsilon_{y}=10$ and $\mathrm{h}=6 \mathrm{~mm}$, an approximately $2.5 \mathrm{~dB}$ reduction of mutual coupling is achieved. An approximately $4.5 \mathrm{~dB}$ reduction of mutual coupling is achieved when we replaced the 2D-EBG with mushroom EBG. Secondly, we studied three high impedance surfaces and applied them to reduce the mutual coupling effect between two arrays patch antennas.. A proposed EBG structure in this work is found to be the most efficient for the reduction of the mutual coupling between $E$-plane coupled antennas. An approximately $6 \mathrm{~dB}$ reduction of mutual coupling is achieved. In H-plane configuration, a $10.8 \mathrm{~dB}$ mutual coupling reduction is achieved at $10.10 \mathrm{GHz}$, which proves that the surface wave is suppressed.

The presence of EBG structure reduces the mutual coupling between the two radiating elements.

\section{REFERENCES}

[1] Ittipiboon, A., Garg, R., Bahl, I., Bhartia, P. Microstrip antenna Design Handbook. Norwood: Artech House, 2000

[2] Jing Liang, and Hung-Yu David Yang, "Radiation Characteristics of a Microstrip Patch over an Electromagnetic Bandgap Surface,"
IEEE Transactions on Antennas and Propagation, Vol. 5, June 2007, pp1691-1697.

[3] E. R. Iglesias, Ó. Q. Teruel, and L. I.Sánchez, "Mutual Coupling Reduction in Patch Antenna Arrays by Using a Planar EBG Structure and a Multilayer Dielectric Substrate," IEEE Trans. Antennas Propag., vol. 56, no. 6, pp. 1648-1655, June 2008..

[4] S. D. Assimonis, T. V. Yioultsis, and Ch. S. Antonopoulos, "Design and Optimization of Uniplanar EBG Structures for Low Profile Antenna Applications and Mutual Coupling Reduction," IEEE Trans. Antennas Propag., vol. 60, no.10, pp. 4944-4949, Oct. 2012.

[5] G.Gnanagurunathan and U.G.Udofia, "Performance analysis of the mushroom-like-EBG structure integrated with a microstrip patch antenna," Proceedings of IEEE Asia-Pacific Conference on Applied Electromagnetic (APACE), 2010.

[6] KOVÁCS, P., RAIDA, Z. Dispersion analysis of planar metallodielectric EBG structures in Ansoft HFSS. In Proc. of the 17th Int.Conf. on Microwaves, Radar and Wireless Communications. Wroclaw (Poland), 2008, p. $1-4$.

[7] L. Liang, C. H. Liang, X. W. Zhao, and Z. J. Su, "A Novel Broadband EBG Using Multi period Mushroom-like Structure," Int. Conf. on Microwave and Millimeter Wave Tech., pp. 16091612, 2008.

[8] Parker and Charlton,: 'Photonic Crystals,' Physics World, 2000.

[9] Saleha A. Shaikh, Ami A. Desai “ Electromagnetic Band Gap Structures in MSA "International Journal of Computer Applications (0975 - 8887) Volume 106 - No.9, November 2014

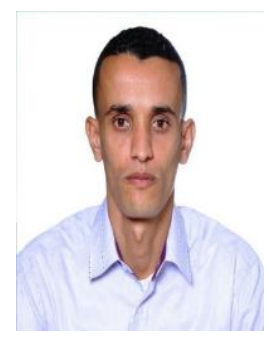

BENYAKHLEF Fethi was born in Remchi, Algeria, in 1986. He received the Majister degree in 2009, from the Abou Bekr Belkaid University in Tlemcen (Algeria). Since 2003, he joined the telecommunication Institute in Tlemcen University (Algeria).

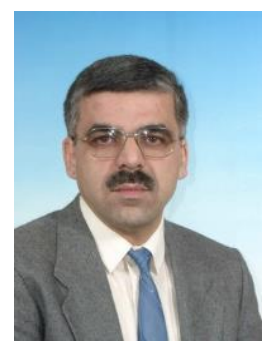

Noureddine Boukli-hacene was born in 1959 in Tlemcen, Algeria. He received his Doctorate Degree (prepared at the "Centre National d'Etudes Spatiale" in Toulouse (France)), in electrical engineering from Limoges university 5France), in 1985 . Then he joined the University of Tlemcen. Since 2012, he is a Professor in electrical engineering at the University of Tlemcen. His research interests include, among others, microstrip antennas and microwave circuits. 